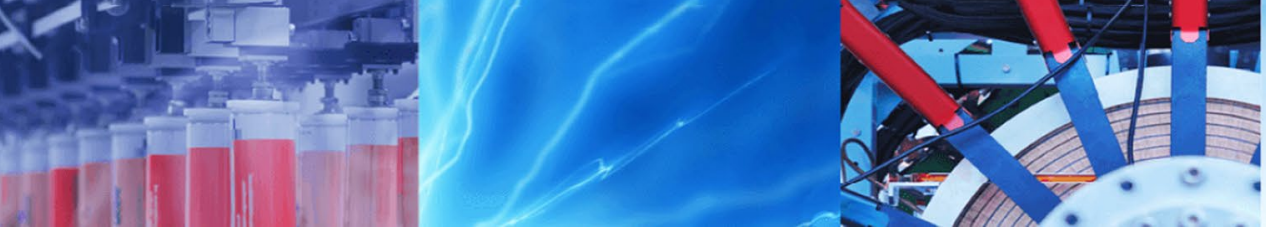

Short Communication

\title{
Structural dynamics of a spinlabeled ribosome elongation factor $P$ (EF-P) from Staphylococcus aureus by EPR spectroscopy
}

\author{
Konstantin S. Usachev ${ }^{1,2}$ D . Evelina A. Klochkova ${ }^{1,2} \cdot$ Alexander A. Golubev $^{1} \cdot$ Shamil Z. Validov $^{1}$. \\ Fadis F. Murzakhanov ${ }^{3}$ - Marat R. Gafurov ${ }^{3}$. Vladimir V. Klochkov ${ }^{2}$ - Albert V. Aganov ${ }^{1}$ - Iskander Sh. Khusainov ${ }^{1,4}$. \\ Marat M. Yusupov ${ }^{1,4}$
}

(c) Springer Nature Switzerland AG 2019

\begin{abstract}
Elongation factor $\mathrm{P}(\mathrm{EF}-\mathrm{P})$ is a three domain protein that binds to the ribosome between $\mathrm{P}$ and $\mathrm{E}$ sites. The EF-P involved in a specialized translation of stalling amino acid motifs such as (PPP or APP). Proteins with stalling motifs are involved in various cell processes, including stress resistance and virulence of bacteria. EF-P stabilizes the P-tRNA and increases the entropy of stalled ribosome complex to compensate for rigid nature of proline residue, thus providing adequate protein synthesis rate. Detailed structural mechanisms of this effect are still poorly understood. It was shown that most of bacteria needs a special post-translational modification in a conservative region of the loop in the domain I of the EF-P located near the CCA-end of P-tRNA and the peptidyl transferase center. In present paper by EPR spectroscopy we investigated a spinlabeled EF-P from Staphylococcus aureus - a pathogenic bacteria which causes various human diseases. Addition of MTSL ((S-(1-oxyl-2,2,5,5-tetramethyl-2,5-dihydro-1H-pyrrol-3-yl)methyl methanesulfonothioate)) label covalently bound to 32Cys in the highly conservative part of EF-P loop in the domain I of the EF-P allows us to analyze protein dynamic by EPR spectroscopy of the high mobility region which was not observed in NMR spectra due to fast proton exchange leading to the absence of the corresponding amide NMR resonances. We suppose that our result could be extended in future for the analysis of EF-P_ribosome complex formation process by advanced EPR techniques.
\end{abstract}

Keywords Elongation factor P.Staphylococcus aureus · Ribosome · EPR · NMR · Chemical exchange

\section{Introduction}

In general, the translation process is produced by ribosomes-complex molecular machines that provide peptide bond synthesis in an accordance with the mRNA nucleotide sequence. The mechanisms of translation in eukaryotes and prokaryotes may be different in many aspects, but fundamentally basic processes are uniform. To ensure an accuracy and an efficiency of the synthesis, in addition to a ribosome, special protein factors are required that are involved at various stages of translation.
In prokaryotes one of such factors is three domain protein elongation factor $\mathrm{P}(\mathrm{EF}-\mathrm{P})$ [1], eukaryotes have a two domain analogue of this factor-elF5A [2]. There is still no integral comprehensive theory that includes the full mechanism of all the functions of EF-P and its regulation.

EF-P have been discovered in 1975 as a stimulating factor during a methionine-puromycin test-its positive effect on the synthesis of the first peptide bond was found [3]. Several studies showed the structure of EF-P in different organisms: Pseudomonas aeruginosa [4], Thermus thermophilus [5]. An important milestone in the development

\footnotetext{
$\triangle$ Konstantin S. Usachev, k.usachev@kpfu.ru| 'Laboratory of Structural Biology, Institute of Fundamental Medicine and Biology, Kazan Federal University, 18 Kremlevskaya, Kazan, Russian Federation 420008. ${ }^{2}$ NMR laboratory, Medical Physics Department, Institute of Physics, Kazan Federal University, 18 Kremlevskaya, Kazan, Russian Federation 420008. ${ }^{3}$ Institute of Physics, Kazan Federal University, 18 Kremlevskaya, Kazan, Russian Federation 420008. ${ }^{4}$ Département de Biologie et de Génomique Structurales, Institut de Génétique et de Biologie Moléculaire et Cellulaire, CNRS, UMR7104, INSERM U964, Université de Strasbourg, 1 rue Laurent Fries, 67400 IIlkirch, France.
} 
of our knowledge about EF-P function was the solution of the EF-P complex structure with the T.thermophilus ribosome [5]. Since then we can say that EF-P is located between $P$ and $E$ sites of the ribosome and is directly adjacent to the P-tRNA. EF-P is a three-domain protein resembling tRNA in shape $[4,6]$. Each of the domains possesses its own role. Most of all is known about the domain I: it is located in vicinity of the CCA-end of P-tRNA and the peptidyl transferase center (PTC). The domain II is in contact with a $\mathrm{L} 1$ protein and the contact is most likely needed to remove the EF-P from the ribosome. The domain III is located near the anticodon loop of P-tRNA, S7 protein and the E-site codon of mRNA. It was proposed that the domain III could play role in frameshift prevention $[7,8]$. Overwhelming number of studies showed that that EF-P provides specialized translation of proteins with polyproline type amino acid stalling motifs $[9,10]$, therefore shift interest from the initiation function of EF-P [5] to the translation function. To maximize the efficiency of the antistalling function, the EF-P needs the special post-translational modification in the conservative region of the loop in the domain I of the EF-P, located close to the PTC (Fig. 1).

There are four known types of possible EF-P PTM of highly conservative loop I of domain I: b-lysinilation [11], rhamnosilation [12], 5-aminopentilation [13] and hypusine in case of elF5A [14]. Approximately one-third of all bacteria possess modifying enzymes corresponding to the known modification [1]. Modification status of other bacteria is unknown. Highly possible modification of S.aureus EF-P could be 5 -aminopentanolitation of $34 \mathrm{~K}$, the same type modification as for B.subtillus EF-P [15].

Bacteria with mutant forms of the EF-P loop, with the absence of EF-P or the absence of EF-P modifying enzymes have phenotypes that can be correlated with specific cell processes such as stress resistance, cell motility and virulence - that could be due to involvement in this processes

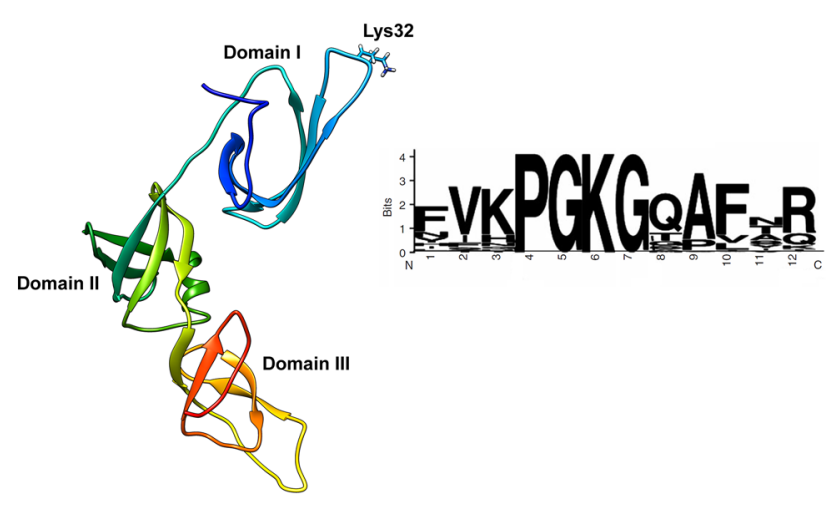

Fig. 1 Structure of elongation factor $P$ (left) and conservative region of EF-P loop from domain I (right) [2]. Side chain is shown as sticks for highly conservative residue Lys 32 which can carry a special post-translational modification proteins containing stalling motifs, regulated by EF-P [2]. Proteins with such sites are often involved in secretion processes, including secretion pathogenicity factors of microorganisms. Last study of EF-P complex with stalled ribosome made by CryoEM [16] proposed some structural mechanism of B-lysinilation modification action for polyproline antistalling, but there are still few details how this modification could work. Also there is no understanding how another types of modification could function and what is the reason for their structural difference.

Therefore the knowledge about dynamics of the loop I could be crucial for understanding of EF-P interaction process with P-tRNA near PTC and the function of modifications. Recently we analyzed a dynamic of SaEF-P backbone amide protons by high-resolution NMR spectroscopy [17] and due to high mobility of the loop between $\beta$-sheets $\beta 2$ and $\beta 3$ (residues $30-34$ ) the amide protons have not been detected in ${ }^{15} \mathrm{~N}-{ }^{1} \mathrm{H}$ heteronuclear NMR spectra due to fast proton exchange leading to the absence of the corresponding amide resonances. Site-directed spin labeling in concert with electron paramagnetic resonance (EPR) spectroscopy is a powerful method for studying the nature of proteins in solution which has been especially useful for examining proteins that are large, flexible or highly dynamic [18-20]. One of the most used spin labeling is achieved by chemically modifying engineered Cys residues with a nitroxide moiety provided by small molecules such as MTSL (Fig. 2). In present paper by EPR spectroscopy we investigated a spinlabeled mutated (K32C) EF-P $(20,5 \mathrm{kDa}$ ) from Staphylococcus aureus (SaEF-P)—a pathogenic bacterium which cause various human diseases and despite the presence of different type of antibiotics is still a significant threat for human health, including a nosocomial infections. The structure of S.aureus EF-P is still absent. The approbation of this method with SaEF-P alone will allow us to check the dynamics of SaEF-P in complex with S.aureus ribosome and, in general, with any other protein that binds to the ribosome.

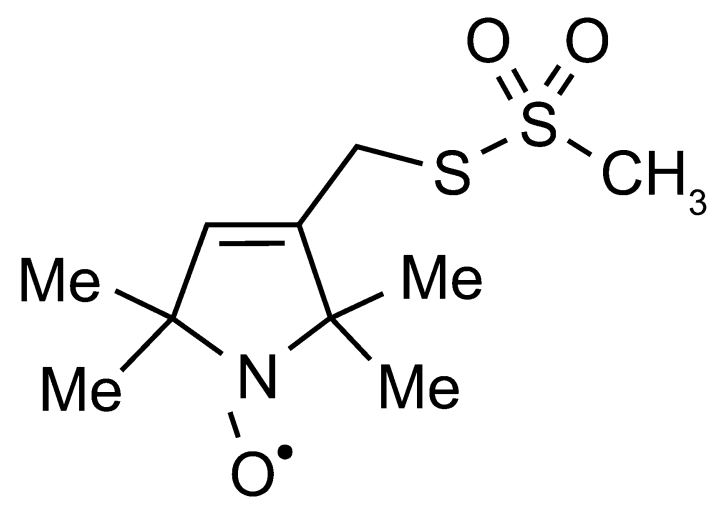

Fig. 2 Chemical structure of nitroxide spin label MTSL 


\section{Materials and methods}

\subsection{Synthesis and purification of EF-P-SL}

Elongation Factor $\mathrm{P}$ from S. aureus (SaEF-P) protein with mutation $\mathrm{K} 32 \mathrm{C}$ and histidine tag at its $\mathrm{C}$-terminus was expressed in Escherichia coli BL21star(DE3) transformed with a pGS21 A plasmid. The cells were dissolved in the lysis buffer ( $20 \mathrm{mM}$ Tris- $\mathrm{HCl}, 200 \mathrm{mM} \mathrm{NH}_{4} \mathrm{Cl}, \mathrm{pH}$ 7.6) with addition of PIC and PMSF. Lisate was clarified by centrifugation at $13.000 \mathrm{rpm}, 4^{\circ} \mathrm{C}$ for $30 \mathrm{~min}$ (Beckman 25.50 rotor). SaEFP-HIS was purified by gravity flow MAC chromatography (Ni-NTA). After a final step of a gel-filtration chromatography on Superdex 75 10/300, the purified protein was dissolved in phosphate buffer $50 \mathrm{mM}$ phosphate buffer $\mathrm{pH}$ 7.4, $250 \mathrm{mM} \mathrm{NH} 4 \mathrm{Cl}$ ).

\subsection{MTSL labeling of EF-P}

MTSL spin label, 2,2,5,5-tetramethyl-1-oxyl-3-methyl methanethiosulfonate (0875000, from Toronto Research Chemicals) was dissolved at $10 \mathrm{mg} / \mathrm{mL}$ concentration in DMSO. For spin labeling, the SaEF-P(K32C) solution was mixed with $\times 10$ ammount of MTSL and incubated $18 \mathrm{~h}$ at $4^{\circ} \mathrm{C}$. The excess spin label was removed by gel exclusion chromatography (gel filtration). Final sample of SaEF-P labeled protein was concentrated to $1 \mathrm{mM}$ with Amicon Ultra Centrifugal Filter Device (10,000 Da cut off) to final volume equal to $500 \mu \mathrm{l}$.

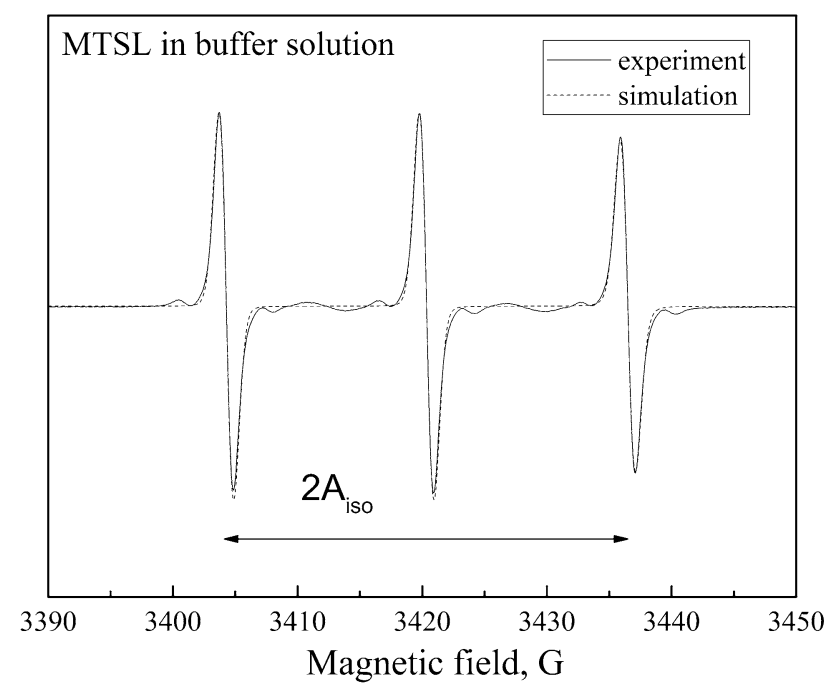

Fig. 3 EPR spectrum of free MTSL spin label in phosphate buffer along with the corresponding simulation with the parameters given in the text and $\tau_{R}=4 \cdot 10^{-11} \mathrm{~S}$

\subsection{Electron paramagnetic resonance spectroscopy}

EPR measurements were done in continuous wave mode by using the abilities of Bruker X-band Elexsys 580 spectrometer at room temperature (Centre of the shared facilities at Kazan Federal University). The solutions were placed into the $0.8 \mathrm{~mm}$ inner diameter quartz tubes. Registration parameters were chosen to be $100 \mu \mathrm{W}$ for the microwave power and $0.2 \mathrm{G}$ at $100 \mathrm{kHz}$ modulation to avoid saturation and overmodulation effects. EPR parameters (isotropic g-factor and hyperfine constant A) and rotational correlation times (by using the values of $\mathrm{g}$ - and $\mathrm{A}$-components for MTSL in water listed in [21] were extracted from the EPR spectra fitting in Easyspin module for Matlab [22].

\section{Results and discussion}

EPR spectrum of MTSL in buffer solution contains 3 lines of hyperfine splitting typical for nitroxide radical in the low-viscous environments caused by the interaction of $\mathrm{S}=1 / 2$ and $\mathrm{I}=1$ for ${ }^{14} \mathrm{~N}$ nuclei (Fig. 3). From experiment parameters $A_{\text {iso }}=16.1 \mathrm{G}, g_{\text {iso }}=2.0059$ in correspondence with the literature data for water solution [21], individual components line widths $\Delta B_{p p}^{+1}=1.13 G, \Delta B_{p p}^{0}=1.12 G$, $\Delta B_{p p}^{-1}=1.15 \mathrm{G}$, can be estimated as well as a correlation time $\tau_{R}=4 \cdot 10^{-11} \mathrm{~s}(40 \mathrm{ps})$.

For spin labeling, the SaEF-P(K32C) solution was mixed with $\times 10$ amount of MTSL and the excess spin label was removed by Gel Exclusion Chromatography and final sample was analyzed by EPR. The results are presented in Fig. 4. The lines broadening due to immobilization, i.e. incomplete averaging by the motion of the $g$-and $A$ component

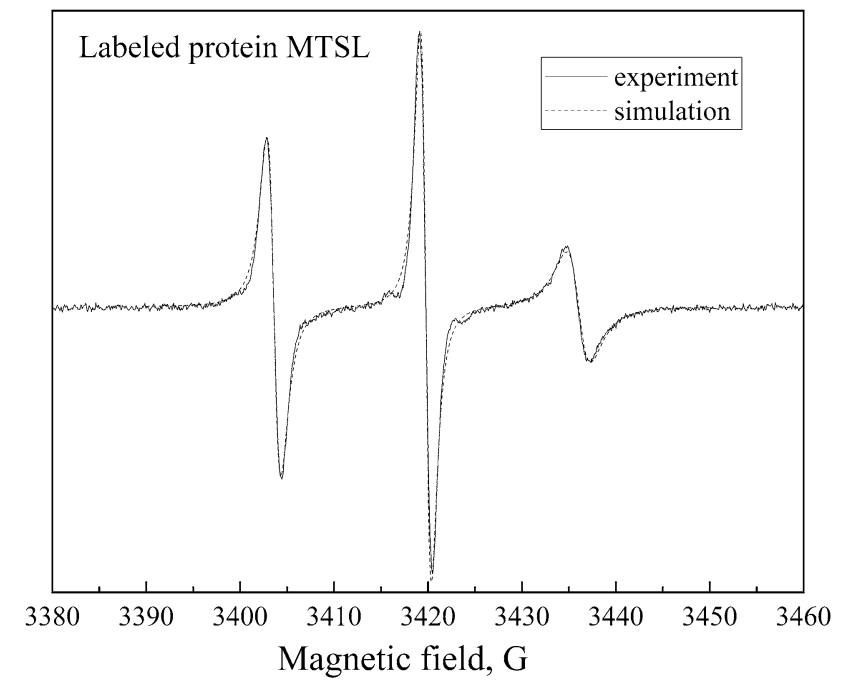

Fig. 4 EPR spectrum MTSL spin label covalently attached to 32Cys with the corresponding simulation with $\tau_{R}=8 \cdot 10^{-10} \mathrm{~s}$ 
$\left(\Delta B_{p p}^{+1}=1.62 G, \Delta B_{p p}^{0}=1.41 G, \Delta B_{p p}^{-1}=2.54 G\right)$ was observed which means that spin label was bound to the protein. Correlation time of MTSL label bound to SaEF-P was estimated as $\tau_{R}=8 \cdot 10^{-10} \mathrm{~s}$.

\section{Conclusion}

Addition of MTSL label covalently bound to 32Cys in the highly conservative part of EF-P loop in the domain I of the EF-P located near the CCA-end of P-tRNA and the peptidyl transferase center (PTC) allows us to analyze protein dynamic by EPR spectroscopy of the high mobility region which was not observed in NMR spectra due to fast proton exchange leading to the absence of the corresponding amide resonances. We believe that our result could be extended in future for the analysis of EF-P_ribosome complex formation process by EPR spectroscopy.

Acknowledgements This work was supported by the Russian Science Foundation (Grant 17-74-20009).

\section{Compliance with ethical standards}

Conflict of interest The authors declare that they have no conflict of interest.

\section{References}

1. Rajkovic $A$, lbba $M$ (2017) Elongation factor $P$ and the control of translation elongation. Annu Rev Microbiol 71:117-131. https:// doi.org/10.1146/annurev-micro-090816-093629

2. Rossi D, Kuroshu R, Zanelli CF, Valentini SR (2014) elF5A and EF-P: two unique translation factors are now traveling the same road. Wiley Interdiscip Rev RNA 5(2):209-222. https://doi.org/10.1002/ wrna.1211

3. Glick BR, Ganoza MC (1975) Identification of a soluble protein that stimulates peptide bond synthesis. Proc Natl Acad Sci U S A 72(11):4257-4260

4. Choi S, Choe J (2011) Crystal structure of elongation factor $P$ from Pseudomonas aeruginosa at 1.75 A resolution. Proteins 79(5):16881693. https://doi.org/10.1002/prot.22992

5. Blaha G, Stanley RE, Steitz TA (2009) Formation of the first peptide bond: the structure of EF-P bound to the $70 \mathrm{~S}$ ribosome. Science 325(5943):966-970. https://doi.org/10.1126/science.1175800

6. Hanawa-Suetsugu K, Sekine S, Sakai H, Hori-Takemoto C, Terada T, Unzai S, Tame JR, Kuramitsu S, Shirouzu M, Yokoyama S (2004) Crystal structure of elongation factor $P$ from Thermus thermophilus HB8. Proc Natl Acad Sci U S A 101(26):9595-9600. https://doi. org/10.1073/pnas.0308667101

7. Gamper HB, Masuda I, Frenkel-Morgenstern M, Hou YM (2015) Maintenance of protein synthesis reading frame by EF-P and $\mathrm{m}(1)$ G37-tRNA. Nat Commun 6:7226. https://doi.org/10.1038/ncomm s8226

8. Alejo JL, Blanchard SC (2017) Miscoding-induced stalling of substrate translocation on the bacterial ribosome. Proc Natl Acad Sci U S A 114(41):E8603-E8610. https://doi.org/10.1073/pnas.17075 39114

SN Applied Sciences
9. Doerfel LK, Wohlgemuth I, Kothe C, Peske F, Urlaub H, Rodnina MV (2013) EF-P is essential for rapid synthesis of proteins containing consecutive proline residues. Science 339(6115):85-88. https:// doi.org/10.1126/science.1229017

10. Peil L, Starosta AL, Lassak J, Atkinson GC, Virumae K, Spitzer M, Tenson T, Jung K, Remme J, Wilson DN (2013) Distinct XPPX sequence motifs induce ribosome stalling, which is rescued by the translation elongation factor EF-P. Proc Natl Acad Sci U S A 110(38):15265-15270. https://doi.org/10.1073/pnas.1310642110

11. Bullwinkle TJ, Zou SB, Rajkovic A, Hersch SJ, Elgamal S, Robinson N, Smil D, Bolshan Y, Navarre WW, Ibba M (2013) (R)-beta-lysine-modified elongation factor $P$ functions in translation elongation. J Biol Chem 288(6):4416-4423. https://doi.org/10.1074/jbc.M112.43887

12. Lassak J, Keilhauer EC, Furst M, Wuichet K, Godeke J, Starosta AL, Chen JM, Sogaard-Andersen L, Rohr J, Wilson DN, Haussler S, Mann M, Jung K (2015) Arginine-rhamnosylation as new strategy to activate translation elongation factor P. Nat Chem Biol 11(4):266-270. https://doi.org/10.1038/nchembio.1751

13. Rajkovic A, Hummels KR, Witzky A, Erickson S, Gafken PR, Whitelegge JP, Faull KF, Kearns DB, Ibba M (2016) Translation control of swarming proficiency in Bacillus subtilis by 5-amino-pentanolylated elongation factor P. J Biol Chem 291(21):10976-10985. https://doi.org/10.1074/jbc.M115.712091

14. Dever TE, Gutierrez E, Shin BS (2014) The hypusine-containing translation factor elF5A. Crit Rev Biochem Mol Biol 49(5):413-425. https://doi.org/10.3109/10409238.2014.939608

15. Witzky A, Hummels KR, Tollerson R 2nd, Rajkovic A, Jones LA, Kearns DB, Ibba M (2018) EF-P Posttranslational Modification Has Variable Impact on Polyproline Translation in Bacillus subtilis. MBio 9(2):100. https://doi.org/10.1128/mbio.00306-18

16. Huter P, Arenz S, Bock LV, Graf M, Frister JO, Heuer A, Peil L, Starosta AL, Wohlgemuth I, Peske F, Novacek J, Berninghausen O, Grubmuller $\mathrm{H}$, Tenson T, Beckmann R, Rodnina MV, Vaiana AC, Wilson DN (2017) Structural basis for polyproline-mediated ribosome stalling and rescue by the translation elongation factor EF-P. Mol Cell 68(3):515-527. https://doi.org/10.1016/j.molcel.2017.10.014

17. Usachev KS, Golubev AA, Validov SZ, Klochkov VV, Aganov AV, Khusainov IS, Yusupov MM (2018) Backbone and side chain NMR assignments for the ribosome elongation factor $\mathrm{P}$ (EF-P) from Staphylococcus aureus. Biomol NMR Assign 12(2):351-355. https ://doi.org/10.1007/s12104-018-9838-z

18. Likhtenshtein Gl, Yamauchi J, Nakatsuji SI, Smirnov Al, Tamura R (2008) Nitroxides: applications in chemistry, biomedicine, and materials science. Wiley, Hoboken. https://doi.org/10.1002/97835 27621743

19. Kokorin $\mathrm{Al}$ (2012) Nitroxides-theory, experiment and applications. Intechopen. https://doi.org/10.5772/2887

20. Kuzhelev AA, Krumkacheva OA, Shevelev GY, Yulikov M, Fedin MV, Bagryanskaya EG (2018) Room-temperature distance measurements using RIDME and the orthogonal spin labels trityl/ nitroxide. Phys Chem Chem Phys 20(15):10224-10230. https:// doi.org/10.1039/c8cp01093e

21. Misra SK (2011) Multifrequency electron paramagnetic resonance. Theory Appl. https://doi.org/10.1002/9783527633531

22. Stoll S, Schweiger A (2006) EasySpin, a comprehensive software package for spectral simulation and analysis in EPR. J Magn Reson 178(1):42-55. https://doi.org/10.1016/j.jmr.2005.08.013

Publisher's Note Springer Nature remains neutral with regard to jurisdictional claims in published maps and institutional affiliations. 